\title{
Aplicación de la norma EN 14491:2006 a los silos de acero cilíndricos para la protección frente a explosiones de polvo
}

\author{
Application of standard EN 14491:2006 to cylindrical steel \\ silos for dust explosion protection
}

A. Tascón $^{(*)}$, P. J. Aguado ${ }^{(*)}$

\section{RESUMEN}

Las explosiones de polvo representan un serio peligro en aquellas industrias y silos en los que se manejan materiales combustibles. Para mitigar los efectos de una posible explosión generalmente se utilizan dispositivos de venteo, que pueden dimensionarse siguiendo la norma europea EN 14491:2006. Sin embargo, frecuentemente surgen complicaciones que hacen que la instalación de venteos sea técnicamente complicada y muy costosa. El objetivo de este trabajo fue analizar la aplicación de la norma EN 14491:2006 a los silos metálicos cilíndricos, remarcando las dificultades y limitaciones existentes y explicando sus aspectos clave para poder aplicarla correctamente. También se ha calculado el coste que puede suponer la protección de silos mediante venteos. Finalmente, se proporcionan algunas sugerencias para afrontar la protección de silos, información sobre métodos alternativos de cálculo de venteos y tendencias de futuro en este campo.

\section{SUMMARY}

Dust explosions represent a serious hazard in industries and silo facilities that handle combustible materials. Venting devices are commonly used to try to mitigate the damage caused by any dust explosion. To calculate vent area size, the recommendations given in European standard EN 14491:2006 can be used. However, the protection of silos is not always simple, and frequently the installation of vents becomes technically difficult and costly. The aim of the present work was to analyse the application of standard EN 14491:2006 to cylindrical steel silos, remarking the existing difficulties and limitations and explaining some critical points in order to understand the aforementioned standard. In addition, the cost of protection by venting in silos was studied. Finally, the authors have provided some suggestions to solve the protection of silos, alternative methods to calculate vent areas and expected future trends in this field.

$760-12$

Palabras clave: Silo; explosión de polvo; venteo; EN 14491; coste.
Keywords: Silo; dust explosion; venting; EN 14491; cost. 


\section{HISTORIA DE LAS EXPLOSIONES DE POLVO}

Cualquier material sólido que pueda reaccionar con el oxígeno del aire lo hará con una violencia y velocidad que crecerán al incrementarse el grado de subdivisión del material. Cuando el producto se encuentre finamente dividido en partículas en suspensión, formando una nube de polvo, el proceso de oxidación puede llegar a tener carácter de explosión. Una explosión de polvo es una reacción exotérmica en cadena en la cual las partículas de polvo reaccionan con el oxígeno del aire a partir de una fuente de ignición que desencadena el proceso, dando lugar a un frente de Ilama que se propaga a través de la nube de polvo (1).

La primera explosión de polvo documentada de la que se tiene noticia ocurrió en 1785 en el almacén de harina de una fábrica de pan en Turín (2). Aunque inicialmente se pensó que la explosión se debió a gases emanados por la harina, la investigación realizada posteriormente evidenció que no se había producido ninguna fermentación, ya que la harina se encontraba con un grado de humedad muy bajo.

A lo largo del siglo XIX, con el incremento extractivo propiciado por la revolución industrial, se sucedieron las explosiones en minas de carbón. Primeramente se responsabilizó de estos fenómenos a la presencia de gases combustibles. Pero en seguida surgieron sospechas sobre la participación del polvo de carbón. El científico inglés Michael Faraday, en 1845, enfatizó el importante papel del polvo de carbón en las explosiones en minas, cuando presentó un informe junto con el geólogo Charles Lyell sobre la explosión de Haswell en 1844, que causó 95 víctimas (2).

En 1910 se creó en Estados Unidos el U.S. Bureau of Mines (USBM), al que se le encomendó, entre otras responsabilidades, impulsar el estudio de las explosiones de polvo para mejorar la seguridad en las minas de carbón. Otros organismos similares surgieron en otros países, como el Safety in Mines Research Board del Reino Unido.

Posteriormente, el estudio de las explosiones de polvo se amplió a otros sectores, siendo caracterizadas una gran cantidad de sustancias potencialmente explosivas en forma de polvo: productos agrarios, metales, plásticos y otras sustancias sintéticas. En la actualidad existen bases de datos exhaustivas de sustancias potencialmente explosivas con sus características más relevantes (3).
A lo largo de la segunda mitad del siglo $X X$ se han Ilevado a cabo grandes esfuerzos para comprender mejor el fenómeno de las explosiones de polvo y especialmente para el desarrollo de medidas que aumentasen la seguridad frente a este tipo de fenómenos (1) (4).

La gran importancia que se concede a la seguridad de los trabajadores en la Unión Europea condujo a la elaboración en los años 90 de dos Directivas sobre atmósferas explosivas, conocidas como Directivas ATEX (ATEX 94/9/CE y ATEX 1999/92/ CE), transpuestas a la legislación española mediante sendos Reales Decretos (5) (6). La Directiva 94/9/CE obliga a los fabricantes a dotar a los equipos que vayan a ser instalados en zonas con atmósferas explosivas con unos determinados requisitos de seguridad. Mientras, la Directiva 1999/92/CE obliga a los empresarios a adoptar medidas técnicas, organizativas y de formación que disminuyan la probabilidad de que ocurra una explosión y que reduzcan sus efectos en caso de que llegue a producirse.

Los materiales que pueden desencadenar una explosión de polvo son: productos orgánicos naturales, propios del sector agroalimentario y forestal (cereales, almidón, azúcar, harina, malta, soja, madera, biomasa, etc.); productos orgánicos sintéticos (plásticos, pigmentos, pesticidas, etc.); metales (aluminio, magnesio, etc.); y carbón. Muchos de estos materiales son con frecuencia almacenados en silos.

\section{EXPLOSIONES DE POLVO EN SILOS}

Un porcentaje considerable de las explosiones de polvo de las que hay registro afectaron a sistemas de almacenamiento. Esto es especialmente cierto en el sector agrario y alimentario, tal y como demostraron las estadísticas recogidas por Schoeff en Estados Unidos (7) o por Jeske y Beck en la República Federal Alemana (8). Algunas de las explosiones se desencadenaron en el interior de un silo. Otras, en cambio, se originaron en equipos o instalaciones anexas, tales como elevadores, ciclones, etc., y posteriormente se propagaron hasta los silos. Las consecuencias de una explosión de polvo en un silo o conjunto de silos pueden ser muy graves, con pérdida de vidas, cuantiosas mermas materiales y económicas, y efectos ambientales indeseables. Ejemplos recientes de explosiones catastróficas de silos son el de Blaye (Francia) en 1997, con 13 muertos, y el de Wichita (USA) en 1998, con 7 muertos; en ambos casos se produjeron daños estructurales muy severos en los silos. 
Según la Directiva ATEX 1999/92/CE, el empresario deberá tomar medidas de carácter técnico y/u organizativo conforme a tres principios básicos, por orden de prioridad: impedir la formación de atmósferas explosivas, evitar la ignición de atmósferas explosivas y atenuar los efectos perjudiciales de una explosión de forma que se garantice la salud y la seguridad de los trabajadores. Estas medidas se combinarán con disposiciones para evitar la propagación de las explosiones. La evaluación de los riesgos de explosión y las medidas adoptadas deben plasmarse obligatoriamente en un Documento de Protección contra Explosiones.

En el interior de los silos es inevitable que se formen nubes de polvo durante los procesos de llenado y vaciado, es decir, atmósferas potencialmente explosivas. En consecuencia, se debe prestar una gran atención a las posibles fuentes de ignición. En muchas situaciones puede no ser suficiente con intentar eliminar las fuentes de ignición y, si así se determina en el Documento de Protección contra Explosiones, se deberán considerar medidas de protección que minimicen los efectos de una posible explosión.

El Eurocódigo 1 ya incluye las cargas debidas a explosiones de polvo como acciones accidentales en silos y depósitos (9) y propone limitar el posible daño mediante la elección de uno o varios de los siguientes métodos de protección: venteo, supresión y diseño resistente. Además plantea el empleo de métodos de confinamiento para evitar la propagación de la explosión de unos equipos a otros.

El sistema de protección más habitualmente empleado en silos es el venteo: una superficie con menor resistencia que el resto de elementos del silo y que se abre a determinada sobrepresión, permitiendo el escape de los gases calientes y limitando la presión máxima dentro del equipo. Los venteos deben situarse en el techo del silo o en las paredes, pero siempre por encima del nivel alcanzado por el material almacenado en su interior. Para grandes volúmenes es el método más sencillo de implementar y en muchos casos el único técnica y/o económicamente viable. En un recinto cerrado sin elementos de venteo la presión generada por una explosión de polvo puede alcanzar valores de 7-10 bar (700-1000 $\mathrm{kPa})$, que provocarían daños estructurales y en muchos casos el colapso del silo.

Los dispositivos de venteo pueden clasificarse en dos tipos en función de su inercia: paneles de venteo, de inercia despreciable, y puertas de explosión, de gran inercia (10).
Mayoritariamente se emplean los paneles de venteo, por su mayor eficacia de venteo y menor mantenimiento. Los paneles de venteo consisten generalmente en una lámina de acero inoxidable. Cuando se produce una explosión el panel se rompe y se deforma sobre sí mismo, dejando un área abierta al exterior. En ocasiones se combina con otras capas muy finas de plástico que proporcionan aislamiento y protección meteorológica e, incluso, condiciones higiénicas para su empleo en la industria alimentaria o farmacéutica. Desde hace años se comercializan diversas tipologías de paneles de venteo, con formas diferentes (rectangulares, circulares, trapezoidales, etc.) y para varias presiones de activación.

La Directiva ATEX 94/9/CE obliga a que los dispositivos de venteo, como sistemas de protección para uso en atmósferas potencialmente explosivas, cumplan determinados requisitos y lleven el correspondiente marcado. Esto sirve para verificar la presión a la que se abre el panel, el área de venteo que proporciona y su seguridad de utilización, ya que un venteo no debe fragmentarse dando lugar a proyectiles.

Sin embargo, la instalación de venteos en silos no siempre es fácil y frecuentemente surgen complicaciones. Especialmente problemáticos son los grandes silos cilíndricos metálicos utilizados en la agricultura y la industria alimentaria, que tienen un techo de muy baja resistencia, lo cual hace que el venteo sea técnicamente complicado y muy costoso, debido a que el área de venteo necesaria aumenta al disminuir la resistencia del recinto a proteger. También es el caso de los silos muy esbeltos, donde el área de venteo necesaria puede llegar a ser mayor que la superficie disponible para instalar venteos.

\section{LA NORMA EUROPEA EN 14491}

En el año 2006 el Comité Europeo de Normalización aprobó la norma europea EN 14491 Dust explosion venting protective systems, que especifica los requisitos para el dimensionamiento de los sistemas de protección por venteo contra explosiones de polvo. Desde noviembre de 2006 ya existe la versión oficial en español (11).

Dicha norma presenta una fórmula empírica para el cálculo de los venteos en recipientes aislados, que se basa en los siguientes parámetros:

- Geometría del recinto a proteger: volumen y relación longitud/diámetro (la longitud es la máxima distancia que podría recorrer la Ilama hasta alcanzar los venteos; en 
el caso de un silo coincide con su altura, ya que los venteos se disponen en el techo o en la parte más superior de la pared) ${ }^{1}$.

- La máxima presión que no queremos que se supere, dependiendo de la resistencia del equipo a proteger (presión de explosión reducida).

- Presión a la que se abrirán los dispositivos de venteo (presión estática de activación).

- Características del polvo: la presión máxima de explosión y el parámetro $K_{s t}$ determinados según métodos normalizados (12) (13).

Todas las presiones que intervienen en el cálculo y a las que se hace referencia en el presente trabajo son presiones relativas, es decir, presiones por encima de la presión atmosférica. La norma UNE-EN 14491:2006 las denomina sobrepresiones.

El área de venteo $A$, para sobrepresiones de 0,1 bar $\leq p_{\text {red }}<1,5$ bar, se calcula como [1]:

$$
A=B(1+C \cdot \log (L / D))
$$

donde $B$ es [2]:

$$
\begin{aligned}
& B=\left\{3,264 \cdot 10^{-5} \cdot p_{\text {máx }} \cdot K_{S t} \cdot p_{\text {red }}^{-0,569}+\right. \\
& \left.+0,27\left(p_{\text {stat }}-0,1\right) \cdot p_{\text {red }}^{-0,5}\right\} \cdot V^{0,753}
\end{aligned}
$$

y $C$ es [3]:

$$
C=\left(-4,305 \cdot \log p_{\text {red }}+0,758\right)
$$

siendo $A$ el área geométrica de venteo $\left(\mathrm{m}^{2}\right)$, $p_{\text {stat }}$ la presión estática de activación (bar), $p_{\text {red }}$ la presión de explosión reducida (bar), $p_{\text {máx }}$ la presión máxima de explosión (bar), $K_{S t}$ la constante de explosión del polvo $(\mathrm{bar} \cdot \mathrm{m} / \mathrm{s}), V$ el volumen del recipiente $\left(\mathrm{m}^{3}\right)$, $L / D$ la relación longitud/diámetro del recipiente (adimensional).

En el caso de sobrepresiones de 1,5 bar $\leq p_{\text {red }} \leq 2,0$ bar se prescinde de la corrección por relación $L / D$ y el cálculo es [4]:

$$
A=B
$$

El área de venteo total requerida $\left(A^{\prime}\right)$, es decir, la que realmente se debe instalar, tendrá en cuenta la eficiencia $\left(\mathrm{E}_{\mathrm{f}}\right)$ del dis-

${ }^{1}$ Si el silo tiene tolva la relación longitud/diámetro es ligeramente reducida según el procedimiento expuesto en el Anexo A de la norma EN 14491:2006.

${ }^{2}$ Los fabricantes de dispositivos de venteo proporcionan un valor nominal de sobrepresión estática de activación (pstat) y un valor de tolerancia, que da lugar a un valor máximo superior de sobrepresión de activación.
La fórmula tiene ciertos límites de aplicación, que se recogen en la Tabla 1 . Hay que resaltar los siguientes aspectos:

- El valor mínimo de $p_{\text {stat }}$ a introducir en la expresión de cálculo debe ser de 0,1 bar, aunque el valor real del dispositivo de venteo sea inferior. Además, el valor que se debe considerar de $p_{\text {stat }}$ es el valor superior del intervalo de tolerancia ${ }^{2}$.

- El método de cálculo propuesto sólo es apropiado para recipientes aislados, es decir, aquellos que están aislados de otros volúmenes mediante dispositivos de aislamiento mecánicos o químicos, no siendo posible la propagación de la explosión de unos equipos a otros.

- La norma no establece para qué intervalo de condiciones iniciales de la nube de polvo es válida. La concentración inicial de la nube de polvo, la velocidad del aire y el nivel de turbulencia condicionan de forma extraordinaria la violencia de la explosión. Sin embargo, estas condiciones son muy distintas según el equipo que se considere, no siendo iguales en un silo, un ciclón o un molino (1). A pesar de ello, la norma no proporciona valores o intervalos de valores de dichos parámetros. Escuetamente menciona que es "válida para la mayoría de aplicaciones prácticas: un recipiente completamente lleno de una nube turbulenta de concentración óptima de polvo", dando a entender que el procedimiento es conservador y queda del lado de la seguridad en la mayoría de las situaciones. Hay que tener en cuenta que en ocasiones no es posible controlar la concentración y turbulencia iniciales; una explosión primaria puede poner en suspensión más polvo e incrementar la turbulencia, dando lugar a explosiones secundarias más violentas.

Los dos métodos más utilizados en todo el mundo para el cálculo de venteos contra explosiones de polvo son la norma europea EN 14491 (11), heredera de la alemana VDI 3673 (14), y la norteamericana NFPA 68 (15). Sin embargo, aunque las dos normas son muy recientes y de reconocido prestigio, conducen a resultados bastante contradictorios en ciertas situaciones. Los trabajos de Tascón et al. (16) tratan de arrojar luz sobre dichas diferencias para el caso de silos, mediante simulaciones CFD (Computational Fluid Dynamics) con el programa DESC (Dust Explosion Simulation Code).

La Figura 1 compara el área de venteo calculada según las dos normas para polvo de granos de soja en un silo de $1000 \mathrm{~m}^{3}$ para una sobrepresión de explosión reducida de 0,2 bar. Dependiendo de la relación altura/diámetro del silo (L/D), el área de venteo requerida según la EN 14491 
Tabla 1. Límites de validez de la fórmula de cálculo propuesta en la norma EN 14491:2006

\begin{tabular}{|c|c|}
\hline Parámetro & Valores límite en EN 14491:2006 \\
\hline Volumen del recipiente $(V)$ & $0,1 \leq V \leq 10.000 \mathrm{~m}^{3}$ \\
\hline $\begin{array}{l}\text { Presión estática de activación del } \\
\text { dispositivo de venteo }\left(p_{\text {stat }}\right)\end{array}$ & $\begin{array}{c}0,1 \leq p_{\text {stat }} \leq 1 \text { bar } \\
\text { para } p_{\text {stat }}<0,1 \text { bar, usar } p_{\text {stat }}=0,1 \text { bar }\end{array}$ \\
\hline Presión de explosión reducida ( $\left.p_{\text {red }}\right)$ & $\begin{array}{c}p_{\text {stat }} \leq p_{\text {red }} \leq 2 \text { bar } \\
\text { se recomienda que } p_{\text {red }} \geq 0,12 \text { bar }\end{array}$ \\
\hline Presión máxima de explosión ( $p_{\text {máx }}$ ) & $\begin{array}{l}5 \leq p_{\text {max }} \leq 10 \text { bar para valores de } K_{s t} \text { de } 10 \mathrm{bar} \cdot \mathrm{m} \cdot \mathrm{s}^{-1} \leq K_{S t} \leq 300 \mathrm{bar} \cdot \mathrm{m} \cdot \mathrm{s}^{-1} \\
5 \leq p_{\text {max }} \leq 12 \text { bar para valores de } K_{s t} \text { de } 300 \mathrm{bar} \cdot \mathrm{m} \cdot \mathrm{s}^{-1} \leq K_{s t} \leq 800 \mathrm{bar} \cdot \mathrm{m} \cdot \mathrm{s}^{-1}\end{array}$ \\
\hline Relación longitud / diámetro $(L / D)$ & $1 \leq L / D \leq 20$ \\
\hline Condiciones atmosféricas & $\begin{array}{c}\text { presión atmosférica entre } 80 \text { y } 110 \mathrm{kPa} \\
\text { temperatura entre }-20{ }^{\circ} \mathrm{C} \text { y } 60{ }^{\circ} \mathrm{C} \\
\text { humedad relativa entre } 5 \% \text { y } 85 \% \text { en volumen } \\
\text { contenido de oxígeno } 20,9 \pm 0,2 \% \text { en volumen }\end{array}$ \\
\hline Otras condiciones & $\begin{array}{c}\text { sin conductos de venteo } \\
\text { eficiencia de venteo }=1 \text { (dispositivos sin inercia) } \\
\text { recipiente aislado }\end{array}$ \\
\hline
\end{tabular}

puede ser significativamente mayor que la recomendada por la NFPA 68, incluso el doble. Las características de explosividad de la $\operatorname{soja}^{3}\left(p_{\text {máx }}=7 \mathrm{bar} ; K_{s t}=73 \mathrm{bar} \cdot \mathrm{m} / \mathrm{s}\right)$ han sido tomadas de los trabajos de Ramírez et al. (17) (18), que recogen las características de explosividad de siete materiales agrícolas muy comunes. Una comparación exhaustiva de las dos normas de venteo aplicadas al caso concreto de los silos fue llevada a cabo por Tascón et al. (19).

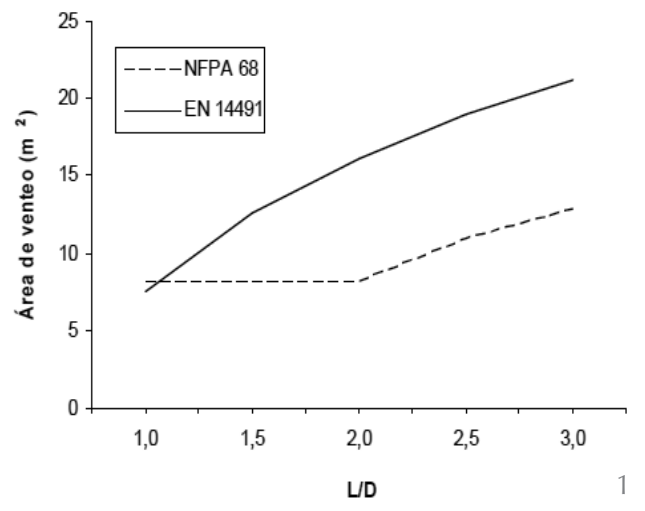

\section{COSTE DE LA PROTECCIÓN DE SILOS MEDIANTE PANELES DE VENTEO}

En el caso de que un equipo, en este caso un silo, deba ser protegido frente a explosiones de polvo mediante dispositivos de venteo porque así se haya determinado en el correspondiente Documento de Protección contra Explosiones, puede resultar de gran interés saber cuál sería el diseño óptimo para reducir el coste que supone su protección. A continuación se presentan algunos criterios, aplicados al caso de silos metálicos cilíndricos, de uso muy habitual en España para el almacenamiento de productos agroalimentarios y agrícolas.

Para poder comparar distintos diseños es conveniente calcular el índice de venteo o densidad de venteo $A_{V}(19)$, cociente entre el área de venteo requerida $(A)$ y el volumen del silo en cuestión ( $V$ ) [6]. De esta manera, se obtienen los $\mathrm{m}^{2}$ de venteo necesarios por cada $\mathrm{m}^{3}$ de almacenamiento de producto y se pueden comparar silos de diferente volumen y geometría.

$$
\text { [6] } \quad A_{V}=A / V
$$

El índice de venteo multiplicado por el precio del metro cuadrado de panel de venteo es igual al coste de protección para $1 \mathrm{~m}^{3} \mathrm{de}$ almacenamiento. Este procedimiento permite incorporar el criterio económico en el diseño de las protecciones contra explosiones y en el diseño de los silos. En la Figura 2 se puede observar el índice de venteo por cada $100 \mathrm{~m}^{3}$ de volumen $\left(A_{V=100}\right)$, calculado según la ecuación [7], siguiendo la norma EN 14491:2006, para diferentes configuraciones de un silo de volumen $1000 \mathrm{~m}^{3}$ para almacenamiento de soja.

$$
\text { [7] } \quad A_{V=100}=A_{V} \cdot 100
$$

Como se deduce de los datos presentados en la Figura 2, la relación $L / D$ tiene una gran influencia en el índice de venteo cuando $p_{\text {red }}$ es baja. Sin embargo, cuando $p_{\text {red }}=1$ bar, la relación $L / D$ apenas tiene influencia en el índice de venteo.

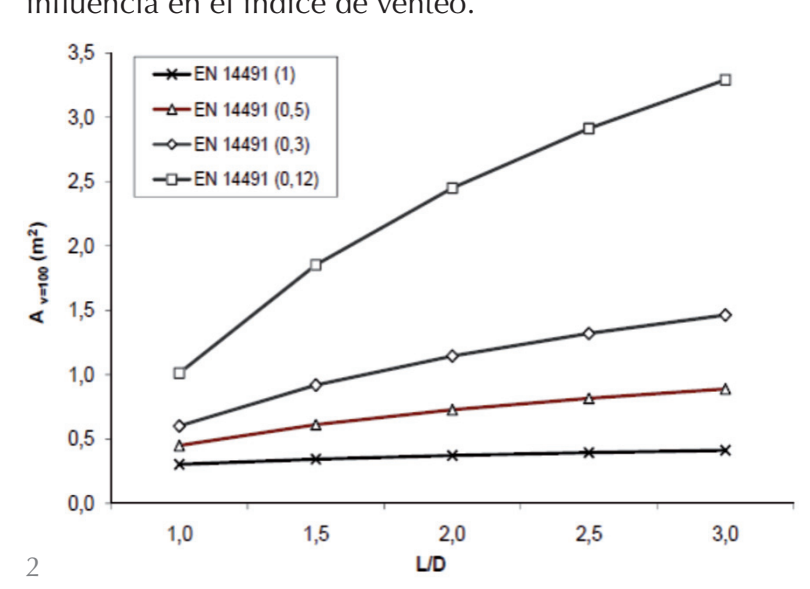

1. Comparación del área de venteo calculada según las normas NFPA 68 (2007) y EN 14491:2006. Soja, silo de 1000 $\mathrm{m}^{3}, p_{\text {red }}=0,2$ bar, $p_{\text {stat }}=0,1$ bar.

2. Índice de venteo $A_{v}$ siguiendo la norma EN 14491:2006 en función de la relación L/D. Soja, silo de 1000 $\mathrm{m}^{3}$, varias $p_{\text {red }}$ (entre paréntesis, en bar).

${ }^{3}$ Los resultados presentados en la Figura 1 han sido calculados para polvo de soja. Para otros productos se obtendrían valores análogos pero cuantitativamente diferentes. 
3. Influencia del volumen del silo sobre el índice de venteo $A_{v=100}$. Áreas de venteo calculadas según EN 14491:2006. Soja, $L / D=1,5, p_{\text {stat }}=0,1$ bar, varias $p_{\text {red }}$
La Figura 3 muestra la influencia del volumen del silo en el índice de venteo para una relación $L / D$ fija, en este caso $L / D=$ 1,5. En el eje $x$ se ha utilizado una escala logarítmica, para facilitar la visualización de un intervalo tan amplio de volúmenes. El índice de venteo aumenta notablemente al disminuir el volumen, especialmente para $p_{\text {red }}$ pequeñas.

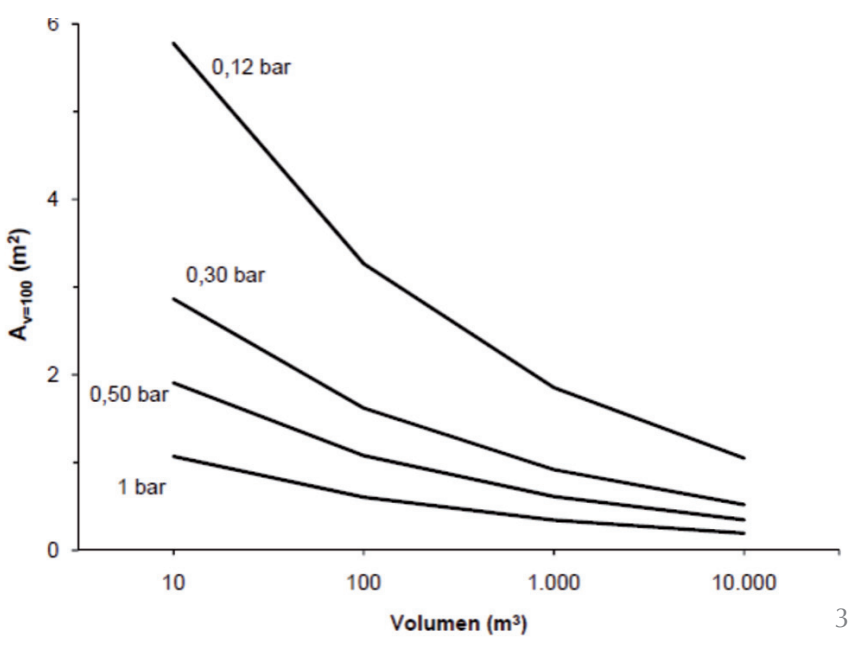

En consecuencia, se pueden extraer dos criterios para el diseño de silos, considerando el coste de la protección mediante venteos. El diseño más eficiente sería:

- Silos de poca esbeltez, con un coste mínimo para $L / D=1$.

- Un número reducido de silos de gran volumen mejor que un mayor número de silos de volumen más pequeño.

Por supuesto, los anteriores criterios no deben de ser considerados aisladamente, sino que deben de sumarse a los criterios que se valoran habitualmente: espacio disponible, coste de la estructura metálica y su montaje, coste de la cimentación, coste de la maquinaria de llenado y vaciado, coste energético de la elevación y trasiego del material, etc.

Sin embargo, si un silo o grupo de silos deben de protegerse mediante venteos, el coste de las protecciones no debe de ser despreciado durante la fase de diseño y concepción del almacenamiento. Para ilustrar con mayor detalle la importancia del coste que supone la protección de silos frente a explosiones con paneles de venteo comerciales, se ha realizado un estudio para algunos ejemplos concretos, empleando para ello información y precios proporcionados por empresas españolas. Aunque los precios considerados son únicamente orientativos y el objeto de este cálculo es más cualitativo que cuantitativo, da una idea bastante clara del incremento en el precio total que supone tener que proteger un silo mediante paneles de venteo.
Los silos considerados en este estudio fueron metálicos, de chapa de acero corrugada y sección circular con fondo plano. El techo era cónico, constituido por placas de forma trapezoidal. Esta tipología de silo es ampliamente utilizada en España. Se han considerado cuatro geometrías distintas. La altura que se ha estimado para determinar $L / D$ es la suma de la altura de la pared cilíndrica del silo más un tercio de la altura del techo cónico, es decir, se ha transformado el cono en una altura de cilindro equivalente con el mismo volumen. Los precios utilizados en el cálculo son precios orientativos de la estructura metálica, calculada para las condiciones más habituales, y no incluye costes de transporte, montaje, IVA, ni tampoco las cimentaciones. Los casos estudiados son los siguientes:

- Silo 1: $V=100 \mathrm{~m}^{3}, L / D=1,3$, precio $=$ $3.200 €$.

- Silo 2: $V=1000 \mathrm{~m}^{3}, L / D=1,3$, precio = $13.100 €$.

- Silo 3: $V=1000 \mathrm{~m}^{3}, L / D=2,5$, precio $=$ $17.300 €$.

- Silo 4: $V=10000 \mathrm{~m}^{3}, L / D=1,2$, precio $=$ $147.000 €$.

Los paneles de venteo se instalarían en el techo, ya que se ha supuesto que no se trataría de un silo aislado, sino de una batería de silos, estando próximos unos de otros. Por otra parte, al instalar los paneles de venteo en el techo se consigue un aprovechamiento máximo del volumen del silo (el material almacenado no debe apoyarse en los paneles de venteo). Se han seleccionado paneles de venteo de acero inoxidable de dimensiones 1492 mm x 450 mm (dimensiones exteriores), con un área de venteo de $0,5217 \mathrm{~m}^{2}$ por panel. Este tipo de panel se ajusta de manera adecuada a las placas que constituyen el techo de este tipo de silos. Su presión de rotura es de 50 mbar ( $\pm 20 \%$ ).

El precio de los paneles de venteo depende de manera muy significativa del número total de paneles encargados. A mayor cantidad de pedido, más reducido es el precio por panel. Por otro lado, cuanto más pequeño es el panel, mayor es el precio por metro cuadrado de área de venteo. El precio del metro cuadrado de panel de venteo puede llegar a variar hasta un $50 \%$ en función de las variables mencionadas.

Como en este estudio se ha supuesto que el silo forma parte de una batería de silos, el número total de paneles necesarios podría presumirse importante (> 50 unidades). Para estas condiciones, el precio del panel, incluyendo la brida correspondiente, se ha estimado en $280 €\left(536,71 € / \mathrm{m}^{2}\right)$ como precio final a usuario. 
Como material almacenado en los silos se ha considerado soja $\left(p_{\text {máx }}=7\right.$ bar; $K_{S t}=$ $73 \mathrm{bar} \cdot \mathrm{m} / \mathrm{s})$.

Las presiones reducidas seleccionadas fueron $p_{\text {red }}=0,30$ bar y $p_{\text {red }}=0,12$ bar; siendo éste último el valor más bajo que aconseja la norma EN 14491:2006 para el cálculo de venteos.

En la Tabla 2 se presenta el procedimiento completo de cálculo del coste para el silo 2, siendo análogo para los otros modelos, e incluye: cálculo del área de venteo según la norma EN 14491:2006, determinación del número de paneles necesarios de forma que el área total de venteo sea igual o superior a la calculada, cálculo del coste total de los paneles, determinación del incremento de coste que supone la protección y el coste total del silo protegido. men de $1.000 \mathrm{~m}^{3}$, se produce un sobrecoste de $2.800 €$ para $p_{\text {red }}=0,3$ bar o de $7.280 €$ para $p_{\text {red }}=0,12$ bar. Por tanto, para un mismo volumen de almacenamiento el diseño geométrico del silo puede variar significativamente el coste derivado de su protección contra explosiones.

En algunos de los casos ( $p_{\text {red }}=0,12$ bar), el número de paneles de venteo a instalar es realmente muy elevado y podrían aparecer dificultades para acomodar un número tan grande de paneles en las placas metálicas trapezoidales que constituyen el techo.

\section{CONSIDERACIONES SOBRE \\ LA PROTECCIÓN DE SILOS FRENTE A EXPLOSIONES}

El venteo no es el único método de protección posible. La supresión (20) es una

Tabla 2.

Coste de la protección del Silo 2 mediante paneles de venteo. Soja, $\mathrm{V}=1000$ m³, L/D = 1,3

\begin{tabular}{|l|c|c|}
\hline Parámetro & $\boldsymbol{p}_{\text {red }}=\mathbf{0 , 3 0}$ bar & $\boldsymbol{p}_{\text {red }}=\mathbf{0 , 1 2}$ bar \\
\hline Área de venteo $\left(\mathrm{m}^{2}\right)$ & 8,07 & 15,56 \\
\hline Índice de venteo $\mathrm{A}_{\mathrm{V}=100}\left(\mathrm{~m}^{2}\right)$ & 0,81 & 1,56 \\
\hline Número de paneles de venteo & 16 & 30 \\
\hline Coste de la protección $(€)$ & 4.480 & 8.400 \\
\hline Coste de la estructura metálica $(€)$ & 13.100 & 13.100 \\
\hline Coste total: estructura + protección $(€)$ & 17.580 & 21.500 \\
\hline Incremento en el coste debido a la protección $(\%)$ & $34,2 \%$ & $64,1 \%$ \\
\hline
\end{tabular}

La Tabla 3 muestra de manera resumida para los cuatro silos estudiados el coste total de los paneles de venteo necesarios y el consiguiente incremento de coste con respecto al precio de la estructura metálica sin venteos. Los resultados muestran que el incremento varía entre el $15 \%$ y el $42 \%$ cuando la presión reducida es de 0,30 bar y entre el $29 \%$ y el $91 \%$ cuando la presión reducida es de 0,12 bar. Por tanto, no es un coste despreciable; muy al contrario, puede representar un sobrecoste realmente importante. alternativa válida si el volumen a proteger es pequeño, aunque puede tener mayores costes de mantenimiento y hay que asegurar la compatibilidad del producto químico supresor con la higiene del proceso en el caso de productos agrícolas, alimentarios o farmacéuticos.

El diseño resistente es otra posibilidad (21). Pero la ausencia total de venteos implica diseñar un silo capaz de resistir la presión máxima que puede desarrollar una explosión de polvo, 7-10 bar para la mayoría de

Tabla 3.

Incremento en el coste de la estructura metálica del silo debido a la protección contra explosiones mediante paneles de venteo. Producto almacenado: soja

\begin{tabular}{|l|c|c|c|c|}
\cline { 2 - 5 } \multicolumn{1}{c|}{} & \multicolumn{2}{c|}{ Coste de los venteos $(\boldsymbol{\epsilon})$} & \multicolumn{2}{c|}{$\begin{array}{c}\text { Incremento en el coste del silo } \\
\text { debido a la protección (\%) }\end{array}$} \\
\hline Tipología de silo & $\mathbf{P}_{\text {red }}=\mathbf{0 , 3 0}$ bar & $\mathbf{P}_{\text {red }}=\mathbf{0 , 1 2}$ bar & $\mathbf{P}_{\text {red }}=\mathbf{0 , 3 0}$ bar & $\mathbf{P}_{\text {red }}=\mathbf{0 , 1 2}$ bar \\
\hline Silo 1 $\left(\mathrm{V}=100 \mathrm{~m}^{3}, \mathrm{~L} / \mathrm{D}=1,3\right)$ & 840 & 1.680 & 26 & 53 \\
\hline Silo 2 $\left(\mathrm{V}=1.000 \mathrm{~m}^{3}, \mathrm{~L} / \mathrm{D}=1,3\right)$ & 4.480 & 8.400 & 34 & 64 \\
\hline Silo 3 $\left(\mathrm{V}=1.000 \mathrm{~m}^{3}, \mathrm{~L} / \mathrm{D}=2,5\right)$ & 7.280 & 15.680 & 42 & 91 \\
\hline Silo 4 $\left(\mathrm{V}=10.000 \mathrm{~m}^{3}, \mathrm{~L} / \mathrm{D}=1,2\right)$ & 22.680 & 42.000 & 15 & 29 \\
\hline
\end{tabular}

Comparando los casos 2 y 3 , si se incrementa la relación longitud/diámetro del silo desde un valor de $L / D=1,3$ a un valor de $L / D=2,5$, manteniendo constante el volu- los productos, lo cual equivale a diseñar un depósito a presión. El diseño resistente se puede considerar inviable económicamente salvo para equipos robustos de pequeño 
4. Presiones perpendiculares a la pared ejercidas por trigo en un silo con tolva a $45^{\circ}$ calculadas mediante un análisis con elementos finitos con el programa ANSYS. Comparación con los valores de $p_{\text {red }}$ de 0,12 bar, 0,30 bar, 0,50 bar y 1 bar. Adaptado de la Tesis de Aguado (21). volumen o aquellos que estén situados en el interior de un edificio y no tengan espacio a donde ventear de forma segura. Sin embargo, el venteo va inevitablemente acompañado de un cierto diseño resistente. Ambos sistemas de protección están inversamente relacionados. A mayor resistencia del equipo, menor área de venteo es necesaria y viceversa. Por tanto, para cada equipo concreto habrá un diseño resistente óptimo que permitirá minimizar el coste total, suma de la estructura metálica y sus protecciones.

No debe olvidarse tampoco que la protección constituye la segunda fase en la lucha contra explosiones. La primera fase es la prevención. En el caso de silos es fundamental prevenir la aparición de posibles fuentes de ignición dentro del silo y en los elementos de transporte anexos. En muchas situaciones será más conveniente y rentable a largo plazo concentrar la inversión en la prevención.

La sobrepresión producida por una explosión de polvo es ejercida sobre todas y cada una de las superficies internas del silo. Ésta es una diferencia fundamental con respecto a otras cargas (empuje del material almacenado, nieve, etc.). Además su magnitud es elevada, aunque se dispongan venteos. Las dos características anteriores consideradas conjuntamente hacen que las acciones debidas a una posible explosión sean determinantes en el diseño de un silo.

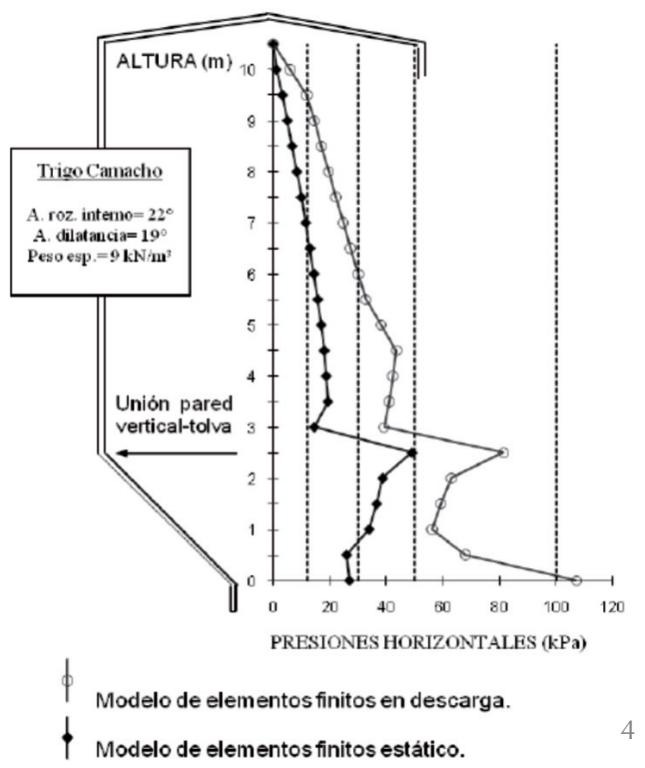

Las cargas ejercidas por el material almacenado en un silo dependen de la geometría del silo, la presencia o no de tolva de descarga, si la situación es de Ilenado o vaciado, el tipo de flujo, las propiedades del material granular, etc. A modo de ejemplo, se comparan en la Figura 4 diferentes valores de $p_{\text {red }}$ con los valores de las presiones perpendiculares a la pared calculadas mediante simulación numérica con elementos finitos para trigo en un silo de $10,5 \mathrm{~m}$ de altura, $6 \mathrm{~m}$ de diámetro y tolva a $45^{\circ}(22)$.

En comparación con las presiones perpendiculares a la pared que son ejercidas por el material almacenado, las presiones de explosión reducidas $\left(p_{\text {red }}\right)$ habitualmente consideradas pueden suponer un valor muy elevado para la parte superior de la pared del silo y, en algunos casos, también para la parte inferior. Para los modelos calculados por Aguado (22), si la presión reducida de explosión se limita a $p_{\text {red }}=0,12$ bar o $p_{\text {red }}=$ 0,30 bar, el silo puede ser capaz de soportar esas presiones sin cambios fundamentales en su diseño, excepto en lo que concierne a la cubierta, que deberá ser calculada de forma específica. Hay que tener en cuenta que la habitual reducción del espesor de la chapa según ascendemos a lo largo de la pared del silo estará condicionada más por el valor seleccionado de $p_{\text {red }}$ que por las acciones ejercidas por el grano.

Para resaltar la magnitud de la carga de explosión aplicada sobre el techo de un silo, basta compararla con la carga de nieve que se considera para el cálculo de edificios en España. Según el Documento Básico "Seguridad Estructural - Acciones en la Edificación" del Código Técnico de la Edificación (23), la carga de nieve por unidad de superficie de proyección horizontal $\left(q_{n}\right)$ para la zona climática más desfavorable y una altitud de 800 m, con un faldón limitado por cornisas sin impedimento al deslizamiento de la nieve y con una inclinación menor o igual a $30^{\circ}$, toma el valor máximo de $1,2 \mathrm{kN} / \mathrm{m}^{2}$. El valor más pequeño de $p_{\text {red }}$ que considera la norma EN 14491:2006 es 0,1 bar $\left(10 \mathrm{kN} / \mathrm{m}^{2}\right)$, que coincide con el valor de $p_{\text {stat }}$ más habitual en los paneles de venteo comerciales. Dicho valor resulta ser más de ocho veces superior a la carga de nieve máxima ${ }^{4}$. Por otro lado, es difícil encontrar en el mercado paneles de venteo que abran a una sobrepresión más baja que 0,05 bar $\left(5 \mathrm{kN} / \mathrm{m}^{2}\right)$, es decir, un valor cuatro veces superior a la carga de nieve anteriormente mencionada.

En consecuencia se puede deducir que muchas de las cubiertas ligeras convencionales que se disponen en los silos metálicos cilíndricos no serían capaces de soportar las presiones de explosión reducidas $\left(p_{\text {red }}\right)$ habitualmente consideradas. Sin embargo, disminuir en exceso la presión reducida $p_{\text {red }}$ para evitar este problema no siempre es posible, ya que al hacerlo aumenta en gran medida el área de venteo requerida. Este problema, asociado a los techos de silos metálicos que se pretende proteger mediante venteos, todavía no está resuelto. 
A juicio de los autores existen dos posibles soluciones a priori. La primera sería diseñar el techo para que funcione como un sistema de venteo. Esta solución implica el desarrollo de una nueva tipología de cubierta, con los correspondientes ensayos y cálculos que permitan asegurar su correcto funcionamiento. Además hay que añadir la dificultad de que todo sistema de protección para uso en atmósferas potencialmente explosivas debe estar certificado y llevar el correspondiente marcado ATEX, según la Directiva 94/9/CE. La segunda solución pasaría por reforzar la cubierta y la parte superior del silo lo necesario para que pueda resistir la sobrepresión de explosión $p_{\text {red }}$ seleccionada e instalar los correspondientes paneles de venteo comerciales, buscando un compromiso entre resistencia del silo y dimensiones del venteo.

Respecto al dimensionamiento de áreas de venteo hay que recalcar que la norma europea EN 14491:2006 no es una norma de obligado cumplimiento, aunque su prestigio y amplia difusión aconsejan que sea tenida en cuenta. Existen otros métodos de dimensionamiento (24). Entre ellos, resulta especialmente conveniente para el caso de silos las fórmulas del Anexo A de la norma alemana VDI 3673 del año 2002 (14), que conducen a áreas significativamente menores para grandes volúmenes (19). Aunque la norma VDI 3673 ha sido la base para la actual norma europea, dichas fórmulas alternativas para silos no fueron incluidas. No obstante, la norma está siendo revisada y mejorada en la actualidad por el panel de expertos del CEN TC 305 WG 3, siendo posible que estas fórmulas alternativas pasen a formar parte de futuras versiones de la norma con el objeto de reducir las dificultades actuales en la protección de silos. Para el dimensionamiento de venteos con inercia en silos se puede recurrir al informe técnico DIN-Fachbericht 140 (25).

Las dimensiones del área de venteo no son el único parámetro a considerar a la hora de implementar este sistema de protección. Aquellos silos protegidos mediante venteos deben de calcularse teniendo en cuenta las fuerzas de reacción que provocaría la expulsión violenta de los gases de combustión a través de los venteos en caso de explosión. Además, cuando se utilicen puertas de explosión - que cierran el área de venteo inmediatamente después de la explosión - hay que considerar la conveniencia de instalar rompedores de vacío para evitar subpresiones provocadas por el brusco enfriamiento de los gases de combustión. La norma EN 14491:2006 incluye ecuaciones para poder estimar ambos fenómenos.
Por otro lado, es fundamental que los venteos se dirijan hacia una zona exterior segura, ya que en el exterior del silo venteado se producirá un incremento de presión y una llamarada. Ambos efectos pueden estimarse cuantitativamente mediante las ecuaciones incluidas en EN 14491:2006 a tal efecto.

Finalmente, es conveniente resaltar el importante papel que pueden desempeñar las simulaciones mediante técnicas CFD (Computational Fluid Dynamics) en el futuro próximo para estimar los efectos de una posible explosión y determinar las mejores configuraciones de los sistemas de prevención y protección. Algunos trabajos basados en estas técnicas ya han sido presentados $(16,26-29)$ y abren un nuevo camino para mejorar la seguridad frente a explosiones. Sin embargo, la confianza en cualquier modelo numérico debe basarse en la validación de los resultados obtenidos mediante ensayos experimentales de explosión.

\section{CONCLUSIONES}

Como resumen del trabajo desarrollado pueden presentarse las siguientes conclusiones:

- El coste que supone la protección mediante venteos es relevante y debe tenerse en consideración desde las primeras fases de diseño de los silos.

- El diseño que minimiza el coste asociado a la instalación de venteos es: silos poco esbeltos $(L / D=1)$ y de gran volumen. - Las acciones ejercidas por una posible explosión, es decir, la presión de explosión reducida $p_{\text {red }}$ resultarán determinantes en el cálculo del techo y la parte superior de la pared del silo.

- En muchos casos el área de venteo exigida por la norma EN 14491:2006 plantea problemas técnicos y un importante coste. - Existen importantes discrepancias entre los diversos métodos de cálculo de venteos. El Anexo A de la norma alemana VDI 3673, del año 2002, proporcionaba un método de cálculo alternativo específico para silos que puede resultar de utilidad en muchas situaciones.

- Ni el cálculo de los silos ni la protección contra explosiones pueden considerarse aisladamente. Ambos problemas están inevitablemente relacionados en aquellas instalaciones con riesgo de explosión.

\section{AGRADECIMIENTOS}

Los autores agradecen a la Consejería de Educación de la Comunidad de Castilla y Léon y al Fondo Social Europeo su apoyo económico a estos trabajos, mediante un proyecto (LE010B05) y una beca de investigación. 


\section{BIBLIOGRAFÍA}

(1) Eckhoff, R. K.: Dust Explosions in the Process Industries, Gulf Professional Publishing/Elsevier, Boston, 2003.

(2) Cardillo, P.: "Some historical accidental explosions". Journal of Loss Prevention in the Process Industries, Vol. 14, n. ${ }^{\circ} 1$ (2001), pp. 69-76. doi:10.1016/S0950-4230(00)00011-5.

(3) BIA-Report 13/97: Combustion and explosion characteristics of dusts, Berufsgenossenschaftliches Institut für Arbeitssicherheit, HVBG, Sankt Augustin, Alemania, 1997.

(4) Bartknecht, W.: Staubexplosionen, Springer-Verlag, Berlin, 1987.

(5) Real Decreto 400/1996, de 1 de marzo, por el que se dicta las disposiciones de aplicación de la Directiva del Parlamento Europeo y del Consejo 94/9/CE, relativa a los aparatos y sistemas de protección para uso en atmósferas potencialmente explosivas, BOE 8 de abril de 1996, Ministerio de Industria y Energía, Madrid, España.

(6) Real Decreto 681/2003, de 12 de junio, sobre la protección de la salud y la seguridad de los trabajadores expuestos a los riesgos derivados de atmósferas explosivas en el lugar de trabajo, BOE 18 de junio de 2003, Ministerio de la Presidencia, Madrid, España.

(7) Schoeff, R.W.: Dust explosion statistics-U.S, Dept. of Grain Science \& Industry, Kansas State University, EEUU, 1984.

(8) Jeske, A.; Beck, H.: "Evaluation of dust explosions in the Federal Republic of Germany". EuropEx Newsletter, n. 9 (1989), pp. 2-4.

(9) EN 1991-4:2006 Eurocode 1, Actions on structures, Part 4, Silos and tanks. European Committee for Standardization, Bruselas, 2006.

(10) García-Torrent, J.: Seguridad industrial en atmósferas explosivas. Laboratorio Oficial J. M. Madariaga, Madrid, 2003.

(11) UNE-EN 14491:2006: Sistemas de protección por venteo de explosiones de polvo. AENOR, Madrid, 2006.

(12) UNE-EN 14034-1:2005: Determinación de las características de explosión de nubes de polvo. Parte 1: Determinación de la presión máxima de explosión $p_{\text {máx }}$ de nubes de polvo. AENOR, Madrid, 2005.

(13) UNE-EN 14034-2:2006: Determinación de las características de explosión de nubes de polvo. Parte 2: Determinación de la velocidad máxima de aumento de presión $(\mathrm{dp} / \mathrm{d} t)_{\text {máx }}$ de nubes de polvo. AENOR, Madrid, 2006.

(14) VDI 3673: Pressure venting of dust explosions. Verein Deutscher Ingenieure. VDI-Verlag GmBH, Düsseldorf, 2002.

(15) NFPA 68: Standard on Explosion Protection by Deflagration Venting. National Fire Protection Association, Quincy, M. A., EE.UU., 2007.

(16) Tascón, A.; Ruiz, Á.; Aguado, P. J.: "Dust explosions in vented silos: Simulations and comparisons with current standards". Powder Technology, Vol. 208, n. 3 (2011), pp. 714-724. doi:10.1016/j. powtec.2011.01.015.

(17) Ramírez, A.; García-Torrent, J.; Aguado, P. J.: "Determination of parameters used to prevent ignition of stored materials and to protect against explosions in food industries". Journal of Hazardous Materials, Vol. 168, n. ${ }^{\circ} 1$ (2009), pp. 115-120. doi:10.1016/j.jhazmat.2009.02.013.

(18) Ramírez, A.; Tascón, A.; García-Torrent, J.: "Experimental determination of self-heating and selfignition risks associated with the dusts of agricultural materials commonly stored in silos". Journal of Hazardous Materials, Vol. 175, n. ${ }^{\circ} 1-3$ (2010), pp. 920-927. doi:10.1016/j.jhazmat.2009.10.096.

(19) Tascón, A.; Aguado, P. J.; Ramírez, A.: "Dust explosion venting in silos: A comparison of standards NFPA 68 and EN 14491". Journal of Loss Prevention in the Process Industries, Vol. 22, n. 2 (2009), pp. 204-209. doi:10.1016/j.jp.2008.12.006.

(20) UNE-EN 14373: Sistemas de supresión de explosiones. AENOR, Madrid, 2006

(21) UNE-EN 14460: Equipos resistentes a las explosiones. AENOR, Madrid, 2006.

(22) Aguado, P. J.: Métodos avanzados de cálculo de presiones en silos agrícolas mediante la técnica de elementos finitos. El vaciado de silos y las paredes de chapa ondulada. Tesis Doctoral. Departamento de Construcción y Vías Rurales, Universidad Politécnica de Madrid, 1996.

(23) CTE DB SE-AE Documento Básico Seguridad Estructural: Acciones en la Edificación. Código Técnico de la Edificación. Ministerio de la Vivienda, 2006.

(24) Abbasi, T.; Abbasi, S. A.: "Dust explosions-Cases, causes, consequences, and control". Journal of Hazardous Materials, Vol. 140. n. ${ }^{\circ} 1-2$ (2007), pp. 7-44. doi:10.1016/j.jhazmat.2006.11.007.

(25) DIN-Fachbericht 140: Design of silos for dust explosions. Beuth-Verlag GmbH, Berlin, 2005.

(26) Wingerden, K. Van; Pedersen, G. H.; Eckhoff, R. K.: "Violence of dust explosions in integrated systems". Process Safety Progress, Vol. 14, n. ${ }^{\circ} 2$ (1995), pp. 131-138.

(27) Zhong, S.; Deng, X.: "Modeling of maize starch explosions in a $12 \mathrm{~m}^{3}$ silo". Journal of Loss Prevention in the Process Industries, Vol. 13 (2000), pp. 299-309.

(28) Kosinski, P.; Hoffmann, A. C.: "An investigation of the consequences of primary dust explosions in interconnected vessels". Journal of Hazardous Materials, Vol. 137, n. ${ }^{\circ} 2$ (2006), pp. 752-761.

(29) Skjold, T.; Arntzen, B. J.; Hansen, O. R.; Storvik, I. E.; Eckhoff, R. K.: "Simulation of dust explosions in complex geometries with experimental input from standardized tests". Journal of Loss Prevention in the Process Industries, Vol. 19, n. ${ }^{\circ} 2-3$ (2006), pp. 210-217. 